\title{
$\ell$-restricted $Q$-systems and quantum affine algebras
}

\author{
Anne-Sophie Gleitz|k
}

Laboratoire de Mathématiques Nicolas Oresme, UCBN, Caen, France

\begin{abstract}
Kuniba, Nakanishi, and Suzuki (1994) have formulated a general conjecture expressing the positive solution of an $\ell$-restricted $Q$-system in terms of quantum dimensions of Kirillov-Reshetikhin modules. After presenting this conjecture, we sketch a proof for the exceptional type $E_{6}$ following our preprint (2013). In types $E_{7}$ and $E_{8}$, we prove positivity for a subset of the nodes of the Dynkin diagram, and we reduce the positivity for the remaining nodes to the conjectural iterated log-concavity of certain explicit sequences of real algebraic numbers.
\end{abstract}

Résumé. Kuniba, Nakanishi et Suzuki (1994) ont formulé une conjecture générale qui exprime la solution positive d'un $Q$-system $\ell$-restreint en fonction des dimensions quantiques de certains modules de Kirillov-Reshetikhin. Après avoir présenté cette conjecture, nous donnons une idée de la preuve pour le type exceptionnel $E_{6}$, selon notre preprint (arXiv, 2013). En types $E_{7}$ et $E_{8}$, nous démontrons la positivité pour certains sommets du diagramme de Dynkin, et nous réduisons la positivité, pour les sommets restants, à une conjecture de log-concavité itérée concernant certaines suites explicites de nombres algébriques.

Keywords: Kirillov-Reshetikhin modules, representation theory, characters, Q-systems, quantum dimension, Kuniba Nakanishi Suzuki (KNS)

\section{$1 \ell$-restricted $Q$-systems}

Let $\delta$ be an ADE Dynkin diagram with vertex set $I$. Kirillov and Reshetikhin [6] have attached to $\delta$ an infinite system of algebraic equations called a $Q$-system, with unknowns $Q_{k}^{(i)}(i \in I, k \in \mathbb{N})$ in a commutative ring. It is given by

$$
\left(Q_{k}^{(i)}\right)^{2}=Q_{k-1}^{(i)} Q_{k+1}^{(i)}+\prod_{j \sim i} Q_{k}^{(j)}, \quad(i \in I, k \geq 1)
$$

where, in the product, the notation $j \sim i$ means that $j$ runs over all neighbours of $i$ in the diagram $\delta$. One usually imposes the initial condition:

$$
Q_{0}^{(i)}=1, \quad(i \in I)
$$

*Email: anne-sophie.gleitz@unicaen.fr

1365-8050 (C) 2014 Discrete Mathematics and Theoretical Computer Science (DMTCS), Nancy, France 


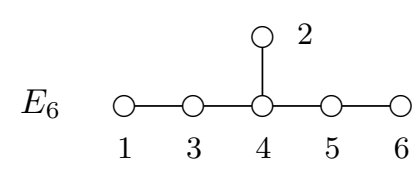

Figure 1: The Dynkin diagram for $\mathfrak{g}=E_{6}$ and its node numbering

Fix a positive integer $\ell$. The $\ell$-restricted $Q$-system has a finite number of unknowns

$$
Q_{k}^{(i)}(i \in I, 0 \leq k \leq \ell)
$$

satisfying the same equations (11) and (2) together with the new boundary condition:

$$
Q_{\ell}^{(i)}=1, \quad(i \in I) .
$$

It is known (see [10]) that $\ell$-restricted $Q$-systems always have a unique positive solution, that is, a unique solution satisfying

$$
Q_{k}^{(i)} \in \mathbb{R}_{>0}, \quad(i \in I, 1 \leq k \leq \ell-1) .
$$

There are various motivations coming from conformal field theory and algebraic $K$-theory to obtain an explicit description of this positive solution [8]. In particular, if $Q_{k}^{(i)}(i \in I, 0 \leq k \leq \ell)$ is this solution, then the numbers

$$
\left.x_{k}^{(i)}:=\frac{\prod_{j \sim i} Q_{k}^{(j)}}{\left(Q_{k}^{(i)}\right)^{2}} \in\right] 0,1[, \quad(i \in I, 1 \leq k \leq \ell-1),
$$

are the arguments of a nice dilogarithm identity, see [8, Th. 5.2, Prop. 14.1].

Example 1.1 Let us write the 2-restricted $Q$-system in type $E_{6}$ (see Figure 1 for the node numbering of the Dynkin diagram). Because of the boundary conditions (1) and (2), there are only 6 unknowns $Q_{i}:=Q_{1}^{(i)}(1 \leq i \leq 6)$, subject to

$$
\left\{\begin{array}{l}
Q_{1}^{2}=1+Q_{3}, \\
Q_{2}^{2}=1+Q_{4}, \\
Q_{3}^{2}=1+Q_{1} Q_{4}, \\
Q_{4}^{2}=1+Q_{2} Q_{3} Q_{5}, \\
Q_{5}^{2}=1+Q_{4} Q_{6}, \\
Q_{6}^{2}=1+Q_{5} .
\end{array}\right.
$$

Because of the unicity of the positive solution, if we further assume that $Q_{i}>0$, we have

$$
Q_{1}=Q_{6}, \quad Q_{3}=Q_{5},
$$

due to the 2-fold symmetry of the Dynkin diagram of $E_{6}$. We are therefore reduced to the system

$$
\left\{\begin{array}{l}
Q_{1}^{2}=1+Q_{3}, \\
Q_{2}^{2}=1+Q_{4}, \\
Q_{3}^{2}=1+Q_{1} Q_{4}, \\
Q_{4}^{2}=1+Q_{2} Q_{3}^{2},
\end{array}\right.
$$


whose unique positive solution is

$$
\left\{\begin{array}{l}
Q_{1}=1+2 \cos \frac{2 \pi}{7} \approx 2.246979604 \\
Q_{2}=\sqrt{2} \cdot \sqrt{\left(4 \cos ^{2} \frac{2 \pi}{7}+6 \cos \frac{2 \pi}{7}+1\right) \cos \frac{2 \pi}{7}} \approx 2.801937735 \\
Q_{3}=8 \cos \frac{2 \pi}{7} \cos ^{2} \frac{\pi}{7} \approx 4.048917341 \\
Q_{4}=\left(2 \cos \frac{2 \pi}{7}+1\right)\left(8 \cos \frac{2 \pi}{7} \cos ^{2} \frac{\pi}{7}-1\right) \approx 6.850855076
\end{array}\right.
$$

If we further set

$$
x_{1}=\frac{Q_{3}}{Q_{1}^{2}}, \quad x_{2}=\frac{Q_{4}}{Q_{2}^{2}}, \quad x_{3}=\frac{Q_{1} Q_{4}}{Q_{3}^{2}}, \quad x_{4}=\frac{Q_{2} Q_{3}^{2}}{Q_{4}^{2}},
$$

we obtain the remarkable identity

$$
2 L\left(x_{1}\right)+L\left(x_{2}\right)+2 L\left(x_{3}\right)+L\left(x_{4}\right)=\frac{6 \pi^{2}}{7},
$$

where $L$ denotes the Rogers dilogarithm function (see e.g. [14]).

\section{The KNS conjecture}

Kuniba, Nakanishi and Suzuki [7] have given a conjectural description of the positive solution of an $\ell$ restricted $Q$-system in terms of quantum dimensions of Kirillov-Reshetikhin modules over the quantum affine algebra attached to $\delta$, which we now recall.

Let $\mathfrak{g}$ be a simple Lie algebra over $\mathbb{C}$ with Dynkin diagram $\delta$. Fix a Cartan subalgebra $\mathfrak{h}$. Let $\Phi \subset \mathfrak{h}^{*}$ be the root system of $\mathfrak{g}$, and $\Phi^{+}$its subset of positive roots. Let $\alpha_{i}, \varpi_{i}(i \in I)$ denote the simple roots and the fundamental weights, respectively. We fix a symmetric bilinear form $(\cdot \mid \cdot)$ on $\mathfrak{h}^{*}$, normalized by

$$
\left(\alpha_{i} \mid \alpha_{i}\right)=2, \quad\left(\alpha_{i} \mid \varpi_{j}\right)=\delta_{i j} .
$$

We denote by

$$
\rho=\sum_{i \in I} \varpi_{i}, \quad \theta=\sum_{i} a_{i} \alpha_{i}
$$

the Weyl vector and the highest root, respectively. Here the $a_{i}(i \in I)$ are the Dynkin labels. We have

$$
(\rho \mid \theta)=\sum_{i \in I} a_{i}=h-1,
$$

where $h$ is the Coxeter number.

Let $P=\bigoplus_{i \in I} \mathbb{Z} \varpi_{i}$ be the weight lattice, and $P_{+}=\bigoplus_{i \in I} \mathbb{N}_{i}$ the monoid of dominant weights. For $\lambda \in P_{+}$, we denote by $V(\lambda)$ the irreducible complex representation of $\mathfrak{g}$ with highest weight $\lambda$, and by $\chi(V(\lambda)) \in \mathbb{Z}[P]$ its character. The dimension of $V(\lambda)$ is given by Weyl's formula:

$$
\operatorname{dim} V(\lambda)=\prod_{\beta \in \Phi^{+}} \frac{(\lambda+\rho \mid \beta)}{(\rho \mid \beta)} .
$$


For $\zeta \in \mathbb{C}^{*}$, and $k \in \mathbb{Z}$ we define the $\zeta$-integer:

$$
[k]_{\zeta}:=\left(\zeta^{k}-\zeta^{-k}\right)\left(\zeta-\zeta^{-1}\right)^{-1}
$$

The $\zeta$-dimension of $V(\lambda)$ is the $\zeta$-analogue of $\operatorname{dim} V(\lambda)$ given by

$$
d_{\zeta}(\lambda):=\prod_{\beta \in \Phi^{+}} \frac{[(\lambda+\rho \mid \beta)]_{\zeta}}{[(\rho \mid \beta)]_{\zeta}} .
$$

When $|\zeta|=1$, this is a real number, well-defined if $[(\rho \mid \beta)]_{\zeta} \neq 0$ for each $\beta \in \Phi^{+}$.

Let $U_{q}(\widehat{\mathfrak{g}})$ be the Drinfeld-Jimbo quantum enveloping algebra of the affine Lie algebra $\widehat{\mathfrak{g}}$ associated with $\mathfrak{g}$ (see e.g. [4]). It has a natural subalgebra isomorphic to the quantum enveloping algebra $U_{q}(\mathfrak{g})$ of $\mathfrak{g}$. Here, we assume that the quantum parameter $q \in \mathbb{C}^{*}$ is not a root of unity. It follows that we can identify the characters of $U_{q}(\mathfrak{g})$ with those of $\mathfrak{g}$.

The Kirillov-Reshetikhin modules $W_{k, a}^{(i)}$ are some special irreducible finite-dimensional representations of $U_{q}(\widehat{\mathfrak{g}})$, depending on three parameters $i \in I, k \in \mathbb{N}, a \in \mathbb{C}^{*}$ (see [8, §4.2]). We will only be interested in their restrictions to $U_{q}(\mathfrak{g})$, which are independent of $a$, and will be denoted by $W_{k}^{(i)}$. The $U_{q}(\mathfrak{g})$-module $W_{k}^{(i)}$ is not irreducible in general. Its character can be expressed as an $\mathbb{N}$-linear combination of irreducible characters of $\mathfrak{g}$ :

$$
\chi\left(W_{k}^{(i)}\right)=\sum_{\lambda \in P_{+}} a_{k}^{(i)}(\lambda) \chi(V(\lambda)) .
$$

Define the $\zeta$-dimension of a Kirillov-Reshetikhin module by

$$
d_{\zeta}\left(W_{k}^{(i)}\right):=\sum_{\lambda \in P_{+}} a_{k}^{(i)}(\lambda) d_{\zeta}(\lambda)
$$

From now on, we fix $\ell \in \mathbb{Z}_{>0}$ and we set

$$
l:=\ell+h, \quad \zeta:=\exp (i \pi / l) .
$$

Because of (7), the $\zeta$-dimension (9) is well-defined for every $\lambda \in P_{+}$.

Conjecture 2.1 ([7, Conjecture 14.2]) The collection of real numbers

$$
Q_{k}^{(i)}:=d_{\zeta}\left(W_{k}^{(i)}\right), \quad(i \in I, 0 \leq k \leq \ell)
$$

is a solution of the $\ell$-restricted $Q$-system. Moreover the following properties hold for any $i \in I$ :

(i) $d_{\zeta}\left(W_{k}^{(i)}\right)=0$ for $k \in \llbracket \ell+1, l-1 \rrbracket$.

(ii) $Q_{k}^{(i)}=Q_{\ell-k}^{(i)}$ for $k \in \llbracket 0, \ell \rrbracket$.

(iii) $Q_{k}^{(i)}>0$ for $k \in \llbracket 0, \ell \rrbracket$.

(iv) $Q_{k}^{(i)}<Q_{k+1}^{(i)}$ for $k \in \llbracket 0,\lfloor\ell / 2\rfloor-1 \rrbracket$. 
Example 2.2 Let us do a quick check in type $A_{3}$ for $\ell=2$. In this simple situation the $U_{q}(\mathfrak{g})$-restrictions of the KR-modules $W_{k}^{(i)}$ are simple, and their dimensions are directly given by 99 . Since $h=4$ we have $\zeta=\exp (i \pi / 6)$, and we easily compute:

$$
\left\{\begin{array}{l}
d_{\zeta}\left(W_{1}^{(1)}\right)=d_{\zeta}\left(\varpi_{1}\right)=\frac{\sin \frac{2 \pi}{3}}{\sin \frac{\pi}{6}}=\sqrt{3}, \\
d_{\zeta}\left(W_{1}^{(2)}\right)=d_{\zeta}\left(\varpi_{2}\right)=\frac{1}{\sin \frac{\pi}{6}}=2, \\
d_{\zeta}\left(W_{1}^{(3)}\right)=d_{\zeta}\left(W_{1}^{(1)}\right)=\sqrt{3}, \\
d_{\zeta}\left(W_{2}^{(1)}\right)=d_{\zeta}\left(2 \varpi_{1}\right)=\frac{\sin \frac{\pi}{2}}{\sin \frac{\pi}{6}} \frac{\sin \frac{2 \pi}{3}}{\sin \frac{\pi}{3}} \frac{\sin \frac{5 \pi}{6}}{\sin \frac{\pi}{2}}=1, \\
d_{\zeta}\left(W_{2}^{(2)}\right)=d_{\zeta}\left(2 \varpi_{2}\right)=\frac{\sin \frac{\pi}{2}}{\sin \frac{\pi}{6}}\left(\frac{\sin \frac{2 \pi}{3}}{\sin \frac{\pi}{3}}\right)^{2} \frac{\sin \frac{5 \pi}{6}}{\sin \frac{\pi}{2}}=1, \\
d_{\zeta}\left(W_{2}^{(3)}\right)=d_{\zeta}\left(W_{2}^{(1)}\right)=1 .
\end{array}\right.
$$

On the other hand, the 2-restricted $Q$-system in type $A_{3}$ reads

$$
\left\{\begin{array}{l}
\left(Q_{1}^{(1)}\right)^{2}=Q_{2}^{(1)}+Q_{1}^{(2)} \\
\left(Q_{1}^{(2)}\right)^{2}=Q_{2}^{(2)}+Q_{1}^{(1)} Q_{1}^{(3)} \\
\left(Q_{1}^{(3)}\right)^{2}=Q_{2}^{(3)}+Q_{1}^{(2)} \\
Q_{2}^{(1)}=Q_{2}^{(2)}=Q_{2}^{(3)}=1,
\end{array}\right.
$$

and it is easy to check that its only positive solution is given by

$$
Q_{1}^{(1)}=Q_{1}^{(3)}=\sqrt{3}, \quad Q_{1}^{(2)}=2 .
$$

So Conjecture 2.1 holds in this case.

\section{Type $E$}

The KNS conjecture is easy to check in type $A$, but it remained an open problem for a long time in other types. Recently, Lee [10] has given a proof of the KNS conjecture in type $D$. We may and will therefore focus hereafter on type $E$.

Let $\mathfrak{g}$ be of exceptional type $E$. We number the nodes of its Dynkin diagram as in Figure 2 Recall that $h=12$ in type $E_{6}, h=18$ in type $E_{7}$, and $h=30$ in type $E_{8}$. In [5] we have proved the following:

Theorem 3.1 The collection of real numbers

$$
Q_{k}^{(i)}:=d_{\zeta}\left(W_{k}^{(i)}\right), \quad(i \in I, 0 \leq k \leq \ell),
$$

is a solution of the $\ell$-restricted $Q$-system. Moreover:

(a) In type $E_{6}$, properties (i), (ii), (iii), (iv) of Conjecture 2.1 hold for any $i \in I$. We also have

$$
d_{\zeta}\left(W_{k+l}^{(i)}\right)=d_{\zeta}\left(W_{k}^{(i)}\right), \quad(k \in \mathbb{N}) .
$$



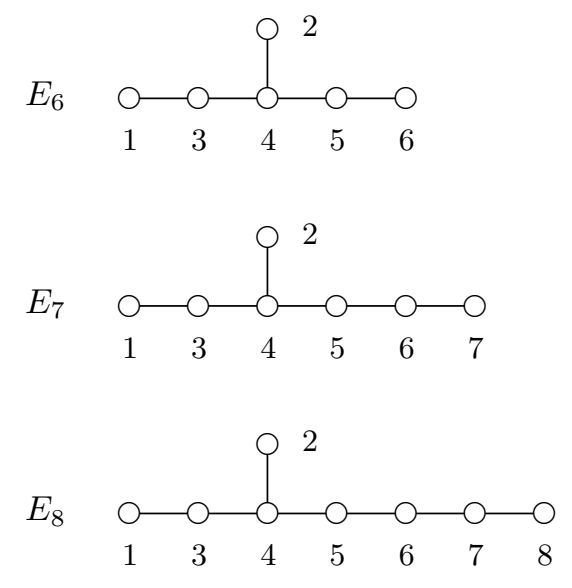

Figure 2: The Dynkin diagrams for $\mathfrak{g}=E_{6}, E_{7}, E_{8}$ and their node numberings

(b) In type $E_{7}$, properties (i), (ii) of Conjecture 2.1 hold for any $i \in I$, property (iii) holds for $i=$ $1,2,3,6,7$, and property (iv) holds for $i=1,2,7$. We also have

$$
\begin{aligned}
& d_{\zeta}\left(W_{k+l}^{(i)}\right)=d_{\zeta}\left(W_{k}^{(i)}\right), \quad(k \in \mathbb{N}, i=1,3,4,6), \\
& d_{\zeta}\left(W_{k+l}^{(i)}\right)=-d_{\zeta}\left(W_{k}^{(i)}\right), \quad(k \in \mathbb{N}, i=2,5,7) .
\end{aligned}
$$

(c) In type $E_{8}$, property ( $i$ ) of Conjecture 2.1 holds for any $i \in I$, property (ii) holds for $i=1,3,4,5,6,7,8$, property (iii) holds for $i=1,3,7,8$, and property (iv) holds for $i=1,8$. We also have for any $i \in I$

$$
d_{\zeta}\left(W_{k+l}^{(i)}\right)=d_{\zeta}\left(W_{k}^{(i)}\right), \quad(k \in \mathbb{N}) .
$$

\section{Outline of proof}

It follows from the Kirillov-Reshetikhin conjecture, proved by Nakajima [12], that the characters $\chi\left(W_{k}^{(i)}\right)(i \in$ $I, k \in \mathbb{N}$ ) give a solution of the unrestricted $Q$-system (1) 2 in the ring $\mathbb{Z}[P]$. The map $\chi(V) \mapsto d_{\zeta}(V)$ being additive and multiplicative, the numbers $d_{\zeta}\left(W_{k}^{(i)}\right)(i \in I, k \in \mathbb{N})$ give therefore a solution of the unrestricted $Q$-system in $\mathbb{R}$. So to prove Conjecture 2.1 we only need to check that

$$
d_{\zeta}\left(W_{\ell}^{(i)}\right)=1 \quad(i \in I)
$$

and that the collection $Q_{k}^{(i)}:=d_{\zeta}\left(W_{k}^{(i)}\right)(i \in I, 0 \leq k \leq l)$ satisfies the additional properties (i), (ii), (iii), (iv).

What makes type $E$ more difficult than type $D$ is that there are many nodes of the Dynkin diagram with labels $a_{i}>2$, and it is difficult to check positivity of the $\zeta$-dimensions at these nodes. Moreover, there are no convenient characters formulas in the literature for the Kirillov-Reshetikhin modules, except for the extremal nodes. To get around these difficulties, we first check the KNS conjecture at the extremal nodes, and then move to the remaining nodes using the $Q$-system. 
Let us outline the strategy of the proof in type $E_{6}$. We start from the following formulas of Chari [3, Section 3]:

$$
\begin{aligned}
\chi\left(W_{k}^{(i)}\right) & =\chi\left(V\left(k \varpi_{i}\right)\right) \quad \text { for } i=1,6, \\
\chi\left(W_{k}^{(2)}\right) & =\sum_{r=0}^{k} \chi\left(V\left(r \varpi_{2}\right)\right) .
\end{aligned}
$$

Using (13) and 99, we can then check rather easily the desired properties at nodes 1 and 6.

Since, by 14 ), $W_{k}^{(2)}$ is not irreducible, we need more tools at node 2. Following Lee [9, 10], we use the level $l$ dot action of the affine Weyl group $\widehat{W}$ of $\widehat{\mathfrak{g}}$ on the weight lattice $P$, denoted by

$$
\lambda \mapsto w \cdot \lambda, \quad(\lambda \in P, w \in \widehat{W}),
$$

see [5]. If $\lambda$ and $w \cdot \lambda$ are dominant, it is known that

$$
d_{\zeta}(\lambda)=(-1)^{\ell(w)} d_{\zeta}(w \cdot \lambda)
$$

where $\ell(w)$ is the length of $w$ in $\widehat{W}$. Applying 15 with $w=s_{0}$ and $\lambda=k \varpi_{2}$, we show that

$$
d_{\zeta}\left(k \varpi_{2}\right)=-d_{\zeta}\left((\ell+1-k) \varpi_{2}\right),
$$

and this formula, together with (14), allows us to check all desired properties at node 2.

Using the $Q$-system equations,

$$
\begin{aligned}
& d_{\zeta}\left(W_{k}^{(1)}\right)^{2}=d_{\zeta}\left(W_{k+1}^{(1)}\right) d_{\zeta}\left(W_{k-1}^{(1)}\right)+d_{\zeta}\left(W_{k}^{(3)}\right), \\
& d_{\zeta}\left(W_{k}^{(2)}\right)^{2}=d_{\zeta}\left(W_{k+1}^{(2)}\right) d_{\zeta}\left(W_{k-1}^{(2)}\right)+d_{\zeta}\left(W_{k}^{(4)}\right), \\
& d_{\zeta}\left(W_{k}^{(6)}\right)^{2}=d_{\zeta}\left(W_{k+1}^{(6)}\right) d_{\zeta}\left(W_{k-1}^{(6)}\right)+d_{\zeta}\left(W_{k}^{(5)}\right),
\end{aligned}
$$

it is then easy to deduce that

$$
d_{\zeta}\left(W_{\ell}^{(3)}\right)=d_{\zeta}\left(W_{\ell}^{(4)}\right)=d_{\zeta}\left(W_{\ell}^{(5)}\right)=1,
$$

and that properties (i) and (ii) are verified at nodes 3, 4, and 5. Moreover, property (3), that is, positivity, is clearly equivalent to the fact that the sequences $d_{\zeta}\left(W_{k}^{(1)}\right), d_{\zeta}\left(W_{k}^{(2)}\right)$ and $d_{\zeta}\left(W_{k}^{(6)}\right)$ are strictly logconcave.

Recall that a finite sequence of real numbers $\left(a_{k}\right)_{0 \leq k \leq n}$ is called log-concave if

$$
a_{k}^{2} \geq a_{k-1} a_{k+1}, \quad(1 \leq k \leq n-1) .
$$

It is strictly log-concave if $a_{k}^{2}>a_{k-1} a_{k+1}$. Using well-known properties of log-concave sequences (see e.g. [13]), we show

Lemma 4.1 For every $i \in I$, the finite sequence $d_{\zeta}\left(k \varpi_{i}\right)\left(0 \leq k \leq \ell / a_{i}\right)$ is strictly log-concave and positive, where $a_{i}$ is the Dynkin label at vertex $i$. 
We then deduce from Lemma 4.1 that $d_{\zeta}\left(W_{k}^{(1)}\right), d_{\zeta}\left(W_{k}^{(2)}\right)$ and $d_{\zeta}\left(W_{k}^{(6)}\right)$ are strictly log-concave, and from this we obtain that properties (iii) and (iv) also hold at nodes $3,4,5$, which finishes the proof for $E_{6}$.

The proofs in type $E_{7}$ and $E_{8}$ are essentially the same as for type $E_{6}$. However log-concavity is no longer sufficient to establish positivity at some inner nodes of the Dynkin diagram, and some stronger form of log-concavity would be necessary. Let us illustrate this in type $E_{7}$.

Following [1], define $\mathfrak{L}$ to be the operator mapping the sequence of real numbers $\left(a_{k}\right)_{0 \leq k \leq n}$ to the sequence $\left(b_{k}\right)_{0 \leq k \leq n}$ defined by

$$
b_{k}:=a_{k}^{2}-a_{k+1} a_{k-1}, \quad(0 \leq k \leq n),
$$

where we have set $a_{-1}=a_{n+1}=0$. Hence $\left(a_{k}\right)$ is log-concave if $\mathfrak{L}\left(a_{k}\right)$ is non-negative. We say that $\left(a_{k}\right)$ is $i$-fold log-concave if the $i$ th iterate $\mathfrak{L}^{i}\left(a_{k}\right)$ is non-negative, and infinitely log-concave if it is $i$ fold $\log$-concave for all $i \in \mathbb{N}$. Interesting examples of $i$-fold log-concave sequences have been studied recently in combinatorics, see e.g. [1, 2, 11].

It is easy to deduce from the $Q$-system that the non-negativity of $Q_{k}^{(i)}$ for $0 \leq k \leq \ell$ and $i=4,5$ amounts to the fact that the sequence

$$
Q_{k}^{(7)}=d_{\zeta}\left(k \varpi_{7}\right), \quad(0 \leq k \leq \ell)
$$

is 3 -fold and 2-fold log-concave. We thus conjecture

Conjecture 4.2 The sequence (17) is $i$-fold log-concave for $i=2,3$.

In fact we believe that this sequence is even infinitely log-concave. Here are a few sample values for $Q_{k}^{(7)}$ when $\ell$ is small.

\begin{tabular}{|c|c|c|c|c|c|c|}
\hline$\ell$ & $Q_{0}^{(7)}$ & $Q_{1}^{(7)}$ & $Q_{2}^{(7)}$ & $Q_{3}^{(7)}$ & $Q_{4}^{(7)}$ & $Q_{5}^{(7)}$ \\
\hline 2 & 1 & 2.2882456111 & 1 & & & \\
\hline 3 & 1 & 3.7912878474 & 3.7912878474 & 1 & & \\
\hline 4 & 1 & 5.4436536231 & 9.0266741836 & 5.4436536231 & 1 & \\
\hline 5 & 1 & 7.1903369448 & 17.142395935 & 17.142395935 & 7.1903369448 & 1 \\
\hline
\end{tabular}

In type $E_{8}$, we state a similar conjecture:

Conjecture 4.3 The sequence

$$
d_{\zeta}\left(W_{k}^{(8)}\right)=\sum_{r=0}^{k} d_{\zeta}\left(r \varpi_{8}\right)
$$

is $i$-fold log-concave for $i=3,4,5$.

This would imply that the sequences $\left(Q_{k}^{(i)}\right)_{0 \leq k \leq \ell}(i=6,5,4)$ are non-negative. 


\section{References}

[1] P. Brändén, Iterated sequences and the geometry of zeroes, J. Reine Angew. Math. 658 (2011), p. $115-131$

[2] W. Chen, D. Dou, A. Yang, Branden's Conjectures on the Boros-Moll Polynomials, arXiv:1205.0305 (2012)

[3] V. Chari, On the fermionic formula and the Kirillov-Reshetikhin conjecture, Int. Math. Res. Notices (2000), p.629-654.

[4] V. Chari and A. Pressley, A guide to quantum groups, Cambridge (1994)

[5] A.-S. Gleitz, On the KNS conjecture in type E, arXiv 1307.2738

[6] A.N. Kirillov and N. Reshetikhin, Representations of Yangians and multiplicities of the inclusion of the irreducible components of the tensor product of representations of simple Lie algebras. J. Sov. Math. 52 (1990), 3156-3164.

[7] A. Kuniba, T. Nakanishi and J. Suzuki, Functional relations in solvable lattice models. I. Functional relations and representation theory. Internat. J. Modern Phys. A 9 (1994), p.5215-5266,

[8] A. Kuniba, T. Nakanishi and J. Suzuki, T-systems and Y-systems in integrable systems, J. Phys. A: Math. Theor. 44 (2011)

[9] C.-H. Lee, Algebraic structures in modular q-hypergeometric series, PhD dissertation, U. C. Berkeley 2012.

[10] C.-H. Lee, A Proof of the KNS conjecture : $D_{r}$ case, arXiv:1210.1669.

[11] P.R. W. McNamara, B. E. Sagan, Infinite log-concavity: developments and conjectures, Adv. Appl. Math. 44 (2010), 1-15.

[12] H. Nakajima, $t$-analogs of $q$-characters of Kirillov-Reshetikhin modules of quantum affine algebras. Represent. Theory 7 (2003), 259-274.

[13] R.P. Stanley, Unimodal and log-concave sequences in algebra, combinatorics, and geometry, in Graph Theory and Its Applications: East and West, Ann. New York Acad. Sci. 576 (1989) pp. 500-535.

[14] D. Zagier, The Dilogarithm function, in Frontiers in Number Theory, Physics and Geometry 2, Springer, 2009. 
